\title{
Automatic Segmentation of Abdominal Adipose Tissue in MRI
}

\author{
Thomas Hammershaimb Mosbech ${ }^{1}$, Kasper Pilgaard ${ }^{2}$, \\ Allan Vaag ${ }^{2}$, and Rasmus Larsen ${ }^{1}$ \\ 1 Technical University of Denmark, DTU Informatics, Richard Petersens Plads, \\ Building 321, DK-2800 Lyngby, Denmark \\ $\{\mathrm{tm}, \mathrm{rl}\} @ i m m . d t u . d k$ \\ 2 Steno Diabetes Center, Niels Steensens Vej 2, DK-2820 Gentofte, Denmark
}

\begin{abstract}
This paper presents a method for automatically segmenting abdominal adipose tissue from 3-dimensional magnetic resonance images. We distinguish between three types of adipose tissue; visceral, deep subcutaneous and superficial subcutaneous. Images are pre-processed to remove the bias field effect of intensity in-homogeneities. This effect is estimated by a thin plate spline extended to fit two classes of automatically sampled intensity points in 3D. Adipose tissue pixels are labelled with fuzzy c-means clustering and locally determined thresholds. The visceral and subcutaneous adipose tissue are separated using deformable models, incorporating information from the clustering. The subcutaneous adipose tissue is subdivided into a deep and superficial part by means of dynamic programming applied to a spatial transformation of the image data. Regression analysis shows good correspondences between our results and total abdominal adipose tissue percentages assessed by dualemission X-ray absorptiometry $\left(R^{2}=0.86\right)$.
\end{abstract}

Keywords: Image processing, MRI, Abdominal adipose tissue, Bias field correction, Tissue classification.

\section{Introduction}

It has been estimated that 171 million people worldwide had diabetes in the year 2000 and that this would increase to 366 million by 2030 [19]. If untreated, diabetes can cause complications such as blindness, amputation and kidney failure. This stresses the importance of methods for screening and early detection.

A measure related to type 2 diabetes is insulin resistance (IR). The gold standard for measuring IR is the hyperinsulinemic euglycemic clamp. However, this method is invasive, expensive and labour intensive - therefore impractical for clinical practice.

Abdominal obesity has a well established association with increased risk of IR and type 2 diabetes. In the assessment of abdominal adipose tissue (AT), it is relevant to distinguish between subcutaneous (SAT) and visceral (VAT), since a strong association between the quantity of VAT and IR is well proven [1].

A. Heyden and F. Kahl (Eds.): SCIA 2011, LNCS 6688, pp. 501 511 2011.

(C) Springer-Verlag Berlin Heidelberg 2011 
Various methods exist for assessing body fat, such as body mass index (BMI), waist size related measures (WS), skin fold callipers, dual-emission X-ray absorptiometry (DXA), X-ray computed tomography (CT) and magnetic resonance imaging (MRI). BMI, WS and callipers only offer crude general approximations, and they (along with DXA) are unable to distinguish between different types of AT, although correlation has been reported [15]. CT can give very detailed information of both quantity and spatial distribution by means of the Hounsfield scale, but the acquisition entails a radiation hazard to the subject. On the other hand, MRI is attractive, as it is a safe modality, also providing visualisations suitable for accurate identification of different AT types.

However, in MRI there is no direct correspondence between intensities and tissue type, and MRI often suffers from problems related to image quality and artefacts. This can call for cumbersome manual or semi-automatic segmentation performed by trained experts. A robust automated assessment from MRI would therefore be highly beneficial in clinics.

A common artefact in MRI is the hardware related bias field; a non-anatomic variability within same-tissue intensity values over the image domain. The inhomogeneity is spatially smooth, with a reported level of variation reaching up to $20 \%$ [18.

One way of correcting the effect is by means of extra images of a uniform phantom acquired in connection with each scan session [11. However, this increases the total scanning time and cost. Instead, we present a method for estimating the effect and correcting the images retrospectively based solely on the MRI of the subject.

Methods from unsupervised learning have successfully been applied for labelling AT by intensity thresholding in MRI. 7] demonstrated the suitability of fuzzy c-means clustering (FCM) for this purpose.

For partitioning the abdomen into the regions containing SAT and VAT, 12. used dynamic programming (DP) coupled with an active shape model, trained on a set of manual segmentations. In [2] a region growing algorithm was used to isolate the VAT. For the same task, a deformable model based on concepts of active contours was applied by [17. Leinhard et al. 16] used atlas-based segmentation, registering a manually segmented prototype to each target image. In our work we combine FCM, DP and active contours in an automatic segmentation method requiring no training data.

It has been suggested to subdivide SAT into superficial SAT (sSAT) and deep SAT (dSAT), anatomically partitioned by Scarpa's fascia (SF) - a thin layer of connective tissue in the abdominal wall. In 14 both dSAT and VAT were found to be strongly associated to IR, whereas sSAT only had a weak relation. Differing from previous methods, we adopt this strategy for our segmentation algorithm, and automatically assess three different types of AT.

\section{Data}

The participants in the study were 40 young healthy twins; 23 men and 17 women, age 18 to 21 years, characterised as ranging from very lean to slightly 
obese with BMI between 14.7 and 21.0. Multi-slice T1 weighted MRI was acquired with a $3 \mathrm{~T}$ Philips Achieva whole body scanner. For each subject, between 15 and 26 slices were used to cover the abdominal region bounded by L1 and L4. Slices with resolution $512 \times 512$ pixels, size $0.8984 \times 0.8984 \mathrm{~mm}^{2}$, thickness $7 \mathrm{~mm}$ and gap $1 \mathrm{~mm}$. For the same region, the total fat percentage was also assessed with DXA.

\section{Methodology}

Our method consists of three parts; a preprocessing step to remove the spatial inhomogeneity of the bias field, a pixel-wise classification identifying AT, and a separation of regions to distinguish between the three types of AT.

\subsection{Bias Field Correction}

A commonly used model for the bias field effect on a voxel with acquired intensity $y_{\text {observed }}$ is the multiplicative link [10]

$$
\begin{aligned}
y_{\text {observed }} & =y_{\text {true }} \cdot y_{\text {bias }} \Leftrightarrow \\
\log \left(y_{\text {true }}\right) & =\log \left(y_{\text {observed }}\right)-\log \left(y_{\text {bias }}\right),
\end{aligned}
$$

where $y_{\text {true }}$ is the true intensity, and $y_{\text {bias }}$ is the bias field effect. With this formulation we can remove the field from an acquired image by subtraction - if the effect is known.

Our method exploits the slow spatial variation and estimates the bias field effect by a smooth function in three dimensions, with parameters obtained through regression.

The regression is based on a set of spatially dense reference voxel points with intensities holding information about the bias field effect. 12 proposed a method based on automatically sampled points corresponding to adipose tissue and the assumption, that these intensities would be similar across the entire image - if not corrupted by the bias field.

However, given the lean nature of the subjects in this study, this method results in too few points, especially from VAT. In order to cover the entire abdomen, we extend the method by regarding two classes of reference points; AT and tissue of high water content.

For each slice, points are sampled automatically across the abdomen as local intensity minima (high water content) and maxima (AT). Under the assumption of a spatially smooth effect, we use $12 \times 9$ rectangles overlapping 10 pixels to subdivide the abdomen. For each rectangle we trim the two point sets to only contain points with intensities within $15 \%$ of each rectangle-minimum and maximum, respectively. To avoid clusters of points, the points are spatially trimmed by $10 \times 10$ non-overlapping rectangles, only keeping the single points in each rectangle with highest and lowest intensity. 
Different values around the chosen, were tested for both the number of rectangles and the percentage thresholds. It was found that they yielded more or less the same sets of points.

After the trimming, the sampled points are gathered from all slices to form a three dimensional point set; $N$ observations $\mathbf{s} \in \mathbb{R}^{3}$, with coordinates $\left[\begin{array}{lll}s_{1} & s_{2} & s_{3}\end{array}\right]^{T}$, $\log$-transformed intensity $y$ and a class-indicator $c$

$$
c(\mathbf{s})= \begin{cases}0 & \text { for } \mathbf{s} \text { high water content } \\ 1 & \text { for } \mathbf{s} \text { adipose tissue }\end{cases}
$$

Supported by the assumption of smooth variation, we use a thin plate spline (TPS) 6 to model the bias field. Estimating this smoothing TPS is formulated as the minimisation a penalised sum of squared differences $S$ subject to the function $f$

$$
S(f)=\sum_{i}^{N}\left\{y_{i}-f\left(\mathbf{s}_{i}\right)\right\}^{2}+\alpha J(f),
$$

where $J(f)$ is the curvature. The parameter $\alpha$ controls the amount of smoothness enforced, our selection of a proper value is described later.

For fitting the two classes of observations, we extend the standard formulation of $f$ presented in 8 ] and 9 ]

$$
f(\mathbf{s})=\beta_{0}+\beta_{1}^{T} \mathbf{s}+\gamma c(\mathbf{s})+\sum_{j}^{n} \delta_{j} h_{j}(\mathbf{s}) .
$$

This way, $\gamma$ is a constant difference between intensities of the two classes.

The basis-functions $h_{j}(\mathbf{s})$ are defined by means of cubed distances from the observation points to $n$ knots $\mathbf{t}$ with coordinates $\left[\begin{array}{ll}t_{1} & t_{2} t_{3}\end{array}\right]^{T}$, located on a regular grid covered by the points.

$$
h_{j}\left(\mathbf{s}_{i}\right)=\left\|\mathbf{s}_{i}-\mathbf{t}_{j}\right\|^{3} .
$$

We formulate (4) as a set of linear equations. The knots and data points are gathered in two coordinate matrices

$$
\mathbf{T}_{k}=\left[\begin{array}{ccc}
1 & \cdots & 1 \\
\mathbf{t}_{1} & \cdots & \mathbf{t}_{n}
\end{array}\right]_{[4 \times n]}, \mathbf{T}_{d}=\left[\begin{array}{ccc}
1 & \cdots & 1 \\
\mathbf{s}_{1} & \cdots & \mathbf{s}_{N}
\end{array}\right]_{[4 \times N]}
$$

The corresponding data values and class-indicators are gathered in two $N \times 1$ vectors $\mathbf{Y}$ and $\mathbf{C}$.

Matrices forming the basis functions of $f$ in (5) are arranged as $\mathbf{E}_{k}$ and $\mathbf{E}_{d}$, with elements computed as

$$
\begin{gathered}
\left\{\mathbf{E}_{k}\right\}_{i j}=h_{j}\left(\mathbf{t}_{i}\right), \text { with } i, j=1, \cdots, n \\
\left\{\mathbf{E}_{d}\right\}_{i j}=h_{j}\left(\mathbf{s}_{i}\right) \text {, with } i=1, \cdots, N \text { and } j=1, \cdots, n
\end{gathered}
$$


Equation (5) can then be written in matrix form

$$
\mathbf{F}=\mathbf{E}_{d} \delta+\mathbf{T}_{d}^{T} \beta+\mathbf{C} \gamma=\left[\mathbf{E}_{d} \mathbf{T}_{d}^{T} \mathbf{C}\right]\left[\begin{array}{l}
\delta \\
\beta \\
\gamma
\end{array}\right]
$$

where the vector $\mathbf{F}$ consists of $N$ elements $\{\mathbf{F}\}_{i}=f\left(\mathbf{s}_{i}\right)$, and $\beta=\left[\beta_{0} \beta_{1}\right]_{[4 \times 1]}$.

Gathering the coefficients $\delta_{j}$ in a $n \times 1$ vector $\delta$, we can write the curvature function $J(f)$ as

$$
J(f)=\delta^{T} \mathbf{E}_{k} \delta
$$

We add a set of linear constraints on the basis function coefficients $\delta_{j}$

$$
\sum_{j=1}^{n} \delta_{j}=\sum_{j=1}^{n} \delta_{j} t_{j 1}=\sum_{j=1}^{n} \delta_{j} t_{j 2}=\sum_{j=1}^{n} \delta_{j} t_{j 3}=0 .
$$

They can be incorporated in the linear system of equations as $\mathbf{T}_{k} \delta=\mathbf{0}$ and a $4 \times 1$ Lagrange multiplier vector $\lambda$.

With this setup (4) can be written as:

$$
\begin{aligned}
S(f)= & {\left[\mathbf{Y}-\mathbf{E}_{d} \delta-\mathbf{T}_{d}^{T} \beta-\mathbf{C} \gamma\right]^{T}\left[\mathbf{Y}-\mathbf{E}_{d} \delta-\mathbf{T}_{d}^{T} \beta-\mathbf{C} \gamma\right] } \\
& +\alpha \delta \mathbf{E}_{k} \delta+\lambda^{T} \mathbf{T}_{k} \delta .
\end{aligned}
$$

We estimate the TPS as the least squares solution to the system of equations, composed by $\frac{\partial S}{\partial \delta}=\frac{\partial S}{\partial \beta}=\frac{\partial S}{\partial \gamma}=\frac{\partial S}{\partial \lambda}=0$,

$$
\left[\begin{array}{cccc}
\mathbf{E}_{d}^{T} \mathbf{E}_{d}+\alpha \mathbf{E}_{k} & \mathbf{E}_{d}^{T} \mathbf{T}_{d}^{T} & \mathbf{E}_{d}^{T} \mathbf{C}^{T} & \mathbf{T}_{k}^{T} \\
\mathbf{T}_{d} \mathbf{E}_{d} & \mathbf{T}_{d} \mathbf{T}_{d}^{T} & \mathbf{T}_{d} \mathbf{C}^{T} & \mathbf{0} \\
\mathbf{C E}_{d} & \mathbf{C} \mathbf{T}_{d}^{T} & \mathbf{C} \mathbf{C}^{T} & \mathbf{0} \\
\mathbf{T}_{k} & \mathbf{0} & \mathbf{0} & \mathbf{0}
\end{array}\right]\left[\begin{array}{c}
\delta \\
\beta \\
\gamma \\
\lambda
\end{array}\right]=\left[\begin{array}{c}
\mathbf{E}_{d}^{T} \mathbf{Y}^{T} \\
\mathbf{T}_{d} \mathbf{Y}^{T} \\
\mathbf{C Y} \mathbf{Y}^{T} \\
\mathbf{0}
\end{array}\right]
$$

From parameter estimates $\hat{\delta}$ and $\hat{\beta}$, the effect $\hat{f}$ can be computed for voxel positions $\mathbf{x}$ gathered in a matrix $\mathbf{T}_{x}$. With a basis function matrix $\mathbf{E}_{x}$ set up as in (9), a vector of the estimated effect $\hat{\mathbf{Y}}_{x}$ with elements $\left\{\hat{\mathbf{Y}}_{x}\right\}_{i}=\hat{f}\left(\mathbf{x}_{i}\right)$ is

$$
\hat{\mathbf{Y}}_{x}=\left[\begin{array}{ll}
\mathbf{E}_{x} & \mathbf{T}_{x}^{T}
\end{array}\right]\left[\begin{array}{l}
\hat{\delta} \\
\hat{\beta}
\end{array}\right]
$$

The effect of a given $\alpha$ to control the rigidity of the TPS varies when fitting to different observation sets. In order to get a consistent estimate across subjects, we adapt a measure of effective degrees of freedom, $d f_{\alpha}[9]$. With this, specifying $d f_{\alpha}=5$ leads to the least squares fitting hyperplane, while $d f_{\alpha}=n$ leads to an interpolating fit.

For the TPS estimation a regular grid of $11 \times 7 \times 5$ knots is used. We find, that a value of $d f_{\alpha}=80$ provides a good overall trade off; fitting the intensity variation caused by the bias field while remaining robust towards noise. While increasing the value to $d f_{\alpha}=120$ does not seem to improve the fit, a choice of $d f_{\alpha}=50$ yields a too rigid hypersurface. Figure 1 illustrates the bias field correction. 

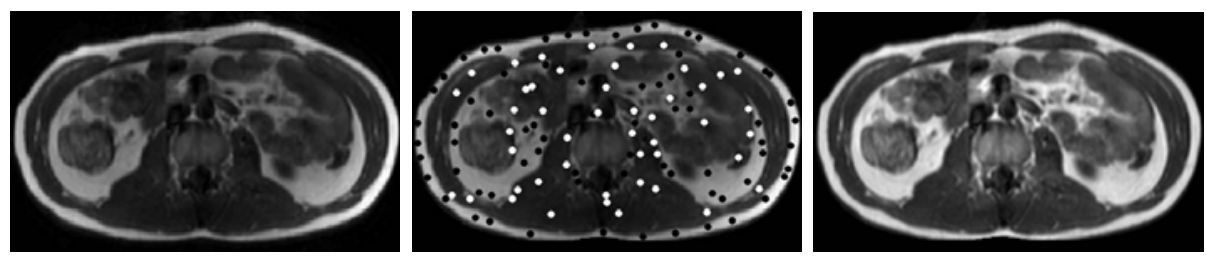

Fig. 1. Bias field correction. Left; the original image. Middle; the two classes of sampled points (black and white are intensity maxima and minima, respectively). Right; the corrected image.

\subsection{Identifying Adipose Tissue}

We label the volume in an unsupervised manner using FCM with random seeds [5], automatically distinguishing between voxels of the two classes by intensity based thresholding.

For a voxel $j$ the FCM algorithm yields membership values $0 \leq u_{j k} \leq 1$, with $k=1,2$ and $u_{j 1}+u_{j 2}=1$, reflecting the degrees of membership to the two clusters. These membership values form a reference between intensity and tissue type comparable between subjects. This way overall determined thresholds on the membership values can adapt to the intensity characteristics in each individual subject. A threshold of $\varepsilon=0.5$, corresponds to labelling as the most likely tissue type.

\subsection{Identifying Regions}

We automatically divide the abdomen into the anatomically defined regions containing dSAT, sSAT and VAT. The region identification is performed slice-byslice.

Active Contours. The SAT is characterized as a ring of homogeneous high intensity located under the skin. The shape of the layer is generally smooth.

The boundary between the SAT layer and the region containing the VAT is located using a variant of the active contours algorithm [1320]. The method is based on spatially evolving a closed curve in 2-dimensions $\mathbf{X}(s)=(X(s), Y(s))$, with parameterisation $s \in[0,1]$. For simplicity, we omit $s$ in the following.

The evolution is driven to minimise the curve energy. By treating the curve $\mathbf{X}$ as a function of time $t$ this corresponds to a dynamic formulation

$$
\gamma \frac{\partial \mathbf{X}}{\partial t}=\mathbf{F}_{\text {int }}(\mathbf{X})+\mathbf{F}_{\text {ext }}(\mathbf{X}) .
$$

The internal force component $\mathbf{F}_{\text {int }}$ should govern the smoothness of the curve during the evolution and make the segmentation robust e.g. towards discontinuities in the image structures of interst. The external force $\mathbf{F}_{\text {ext }}$ should incorporate image information related to the segmentation task. $\gamma$ merely makes the units on both sides consistent. 
The internal force is written as

$$
\mathbf{F}_{\text {int }}(\mathbf{X})=\frac{\partial}{\partial s}\left(\alpha \frac{\partial \mathbf{X}}{\partial s}\right)-\frac{\partial^{2}}{\partial s^{2}}\left(\beta \frac{\partial^{2} \mathbf{X}}{\partial s^{2}}\right),
$$

where $\frac{\partial \mathbf{X}}{\partial s}$ is the degree of stretching, while $\frac{\partial^{2} \mathbf{X}}{\partial s^{2}}$ is the curvature. The contributions of the two are controlled by weights $\alpha$ and $\beta$.

We use a balloon model [3, forming the external force by two terms

$$
\mathbf{F}_{\mathrm{ext}}(\mathbf{X})=\mathbf{F}_{\mathrm{def}}(\mathbf{X})+\mathbf{F}_{\mathrm{imp}}(\mathbf{X})
$$

where $\mathbf{F}_{\text {def }}$ is a deflation force, and $\mathbf{F}_{\mathrm{imp}}$ is an impurity force.

The deflation force for a point $\mathbf{X}$ on the curve is defined as

$$
\mathbf{F}_{\mathrm{def}}(\mathbf{X})=w_{\mathrm{def}} \mathbf{N}(\mathbf{X})
$$

where $w_{\text {def }}>0$ is a weighting parameter, and $\mathbf{N}(\mathbf{X})$ is the inward unit normal for the curve at the point. A deformation using $\mathbf{F}_{\text {def }}$ alone would cause the curve to contract - like a deflating balloon.

The second force term of (18), $\mathbf{F}_{\mathrm{imp}}$, is directed opposite of $\mathbf{F}_{\mathrm{def}}$, and should neutralise the deformation by means of image information.

In the formulation of $\mathbf{F}_{\mathrm{imp}}$, we rely on the assumption of distinct homogeneous high intensities in the SAT layer. At a given time in the deformation, the force magnitude is defined by the content of the region enclosed by the initial and current curve.

The magnitude is zero for a pure area, and is designed to grow proportional to the share of impure intensity covered. For a point $\mathbf{X}$ on the curve, we write

$$
\mathbf{F}_{\mathrm{imp}}(\mathbf{X})=-w_{\mathrm{imp}} \mathbf{N}(\mathbf{X}) \int_{0}^{1} H\left(\Theta-I\left(\mathbf{X}_{z}\right)\right) d z,
$$

with weight $w_{\text {imp }}>0$. $H$ is a Heaviside step function based on a threshold $\Theta$ and image value $I$ at the location $\mathbf{X}_{z}=(1-z) \mathbf{X}_{\text {init }}+z \mathbf{X}$. That is, the line integral between the point position on the initial and current curve.

To define $\Theta$ in a generic fashion across subjects, we base the segmentation on the AT-class membership values provided by the FCM algorithm. This way the intuitive choice $\Theta=0.5$, gives good results.

The method is first used for segmenting the outer boundary of the SAT layer; initialised as a circle around the abdomen, with impurity based on non-AT. This is then used to initialise the inner boundary segmentation. Figure 2 shows an example of the SAT-layer segmentation.

Dynamic Programming. We partition the SAT layer into a deep and a superficial part by identifying SF. On the MRI, SF appears as a smooth low intensity line on the high intensity AT. The shape generally follows the outline of the abdomen. We make use of these properties in the segmentation; spatially transforming the image and applying dynamic programming (DP) [4/5]. 


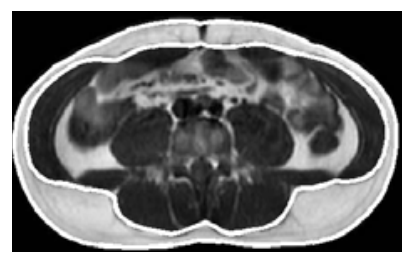

Fig. 2. SAT-layer segmentation by active contours
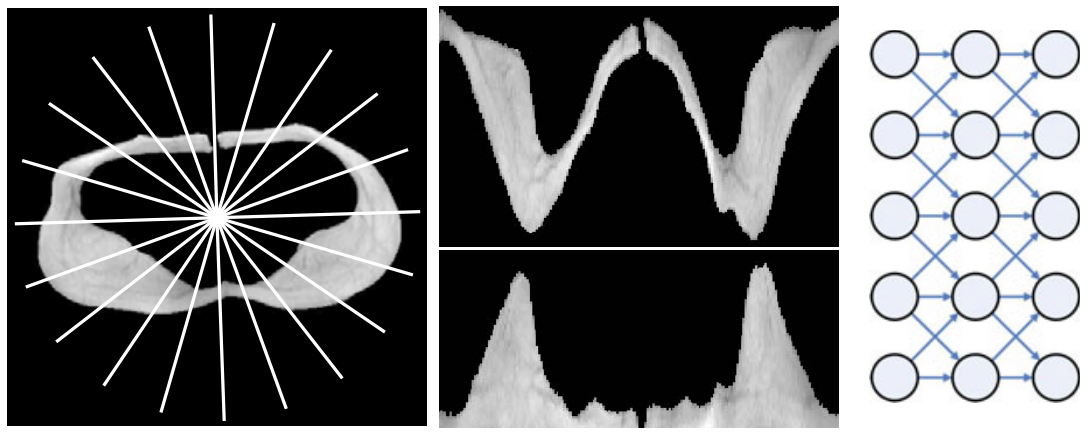

Fig. 3. The spatial transformation of the SAT layer. Left; 20 profiles illustrating the resampling concept. Middle; the resampled image using 200 profiles and the flat transformed image. Right; directed acyclic graph illustrating the edge structure.
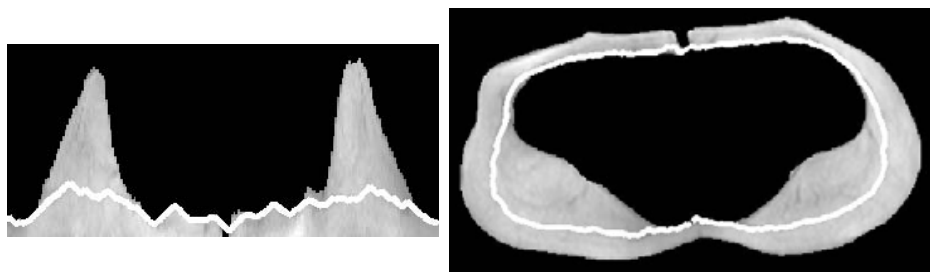

Fig. 4. Partitioning of the SAT layer by DP. Left; transformed image. Right; original set-up.

We apply a polar transformation of the SAT layer; resampling along profiles from the centre. Furthermore, to make SF appear straighter, the resampled image is flattened removing all zero-intensity pixels outside the SAT-layer. Figure 3 illustrates the resampling steps.

In the transformed image, SF runs approximately straight across the image. We utilise this, and regard the image as a directed acyclic graph; pixels are vertices weighted by their intensity with connecting directed edges as illustrated on the rightmost drawing on Fig. 3 ,

This way, we can identify SF as the shortest path from a vertex in the leftmost column to one in the rightmost. The edge structure chosen is suitable, as it 
enforces smoothness on the path. Figure 4 shows the resuling path on both the transformed image and the original set-up - after transforming it back.

\section{Results}

The clustering and the identified regions are combined to form the final segmented volume. We observe a slight difference in the characteristics of VAT and SAT, as the MRI generally exhibit less contrast for the latter, due to the partial volume effect. We handle this by means of locally defined thresholds; $\varepsilon=0.85$ for VAT and $\varepsilon=0.50$ in the SAT layer. Figure 5] shows an example of the final segmentation.

The segmented volumes were subject to a visual inspection by medical experts. On slices, where blurry artefacts in the anterior part were caused by insufficient breath-holding during image acquisition, the SAT layer identification generally proved robust.

Most subjects featured very little anterior SAT. A decision was therefore made to only consider the subdivision in the posterior. On slices with little SAT, SF coincides with the inner SAT layer boundary (e.g. Fig. 1). Here, the DP scheme classified the entire layer as sSAT.
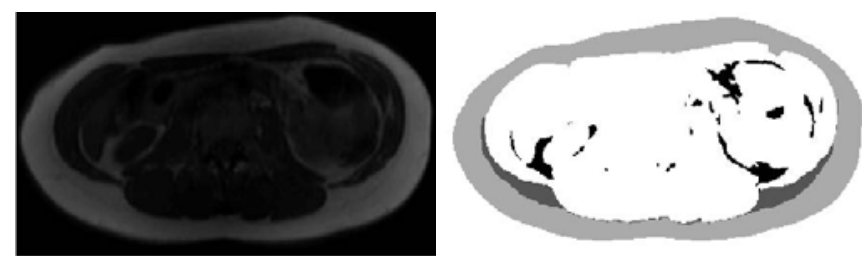

Fig. 5. Final AT segmentation. Light grey; sSAT. Dark grey; dSAT. Black; VAT.

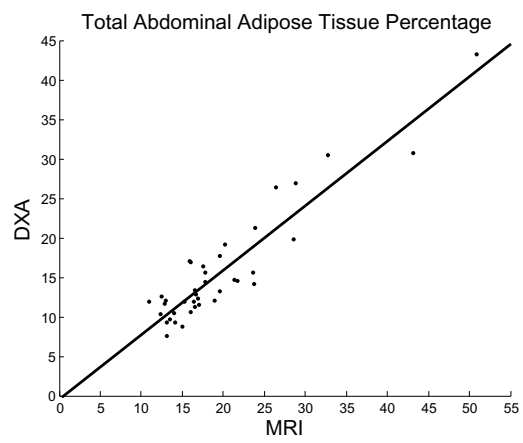

Fig. 6. Corresponding percentages of total abdominal adipose tissue; computed with our MRI-based method and assessed by DXA. The line shows the linear regression fit $y=-0.3975+0.8169 x$ with $R^{2}=0.86$. 
As a quantitative assessment, the obesity was reported as three volume percentages for each subject; sums of voxels in each AT-class relative to the total sum of voxels in the abdomen.

Figure 6 shows a comparison of our total AT percentage with a corresponding measure assessed by DXA. A linear regression analysis showed good a fit between the two $\left(y=-0.3975+0.8169 x, R^{2}=0.86\right)$. Our method seems to produce slightly higher values in comparison to DXA-measurements. This supports our choice of applying a stricter threshold for VAT, as this over-estimation would have been more distinct.

\section{Conclusion}

This work presents a method for fully automated segmentation of VAT, dSAT and sSAT in abdominal MRI.

The method automatically performs a three-dimensional correction of the MRI to remove the hardware-related bias field effect, enabling intensity based tissue classification.

The unsupervised classification scheme, allows us to use structures in intensity distributions related to the two classes. Furthermore, we can distinguish between characteristics of VAT and SAT.

The deformable model identifying the SAT layer proved to handle discontinuities and artefacts well without a training set. Our inclusion of image information uses results from the tissue classification, adapting to the individual subjects to makie the segmentations more robust.

Considering that both quantities of VAT and dSAT have a reported connection to IR, our partitioning into sSAT and dSAT has an advantage over VAT due to the degree of partial volume effect present in the VAT on the MRI used in this study. Where the appearance of SF generally is well-defined; either clearly visible on the SAT layer or coinciding with the interior SAT boundary.

The segmentations were visually validated by medical experts. Furthermore, to evaluate the tissue classification, total percentages of AT measured by our method was compared to similar measures obtained from DXA of the same abdominal region. A linear regression showed good correspondence $\left(R^{2}=0.86\right)$.

The developed automated segmentation method proved suitable for distinguishing between three types of abdominal AT from MRI. In comparison to manual and semi-automatic segmentations, our method enables a much larger throughput and eliminates intra- and interobserver variability.

\section{References}

1. Abate, N., Garg, A., Peshock, R.M., Stray-Gundersen, J., Grundy, S.M.: Relationships of generalized and regional adiposity to insulin sensitivity in men. Journal of Clinical Investigation 96(1), 88 (1995) 
2. Armao, D., Guyon, J.P., Firat, Z., Brown, M.A., Semelka, R.C.: Accurate quantification of visceral adipose tissue (VAT) using water-saturation MRI and computer segmentation: Preliminary results. Journal of Magnetic Resonance Imaging 23(5), 736-741 (2006)

3. Cohen, L.D.: On active contour models and balloons. CVGIP: Image Understanding 53(2), 211-218 (1991)

4. Cormen, T.H., Leiserson, C.E., Rivest, R.L., Stein, C.: Introduction to Algorithms, 2nd edn. MIT Press, Cambridge (2001)

5. Dawant, B.M., Zijdenbos, A.P.: Handbook of Medical Imaging. Medical Image Processing and Analysis, ch.2, vol. 2. SPIE Press, Bellingham (2000)

6. Duchon, J.: Interpolation of functions of two variables following the principle of the bending of thin plates. Revue Francaise d'Automatique Informatique Recherche Operationnelle 10, 5-12 (1976)

7. Engholm, R., Dubinskiy, A., Larsen, R., Hanson, L.G., Christoffersen, B.Ø.: An adipose segmentation and quantification scheme for the abdominal region in minipigs. In: International Symposium on Medical Imaging 2006, The International Society for Optical Engineering. SPIE, San Diego (February 2006)

8. Green, P.J., Silverman, B.W.: Nonparametric Regression and Generalized Linear Models: A Roughness Penalty Approach. Chapman and Hall, Boca Raton (1994)

9. Hastie, T., Tibshirani, R., Friedman, J.H.: The Elements of Statistical Learning. Springer, Heidelberg (2001)

10. Hou, Z.: A review on MR image intensity inhomogeneity correction. International Journal of Biomedical Imaging 2006, 1-11 (2006)

11. Ji, Q., Glass, J.O., Reddick, W.E.: A novel, fast entropy-minimization algorithm for bias field correction in $\mathrm{mr}$ images. Magnetic Resonance Imaging 25, 259-264 (2007)

12. Jørgensen, P.S., Larsen, R., Wraae, K.: Unsupervised assessment of subcutaneous and visceral fat by MRI. In: Salberg, A.-B., Hardeberg, J.Y., Jenssen, R. (eds.) SCIA 2009. LNCS, vol. 5575, pp. 179-188. Springer, Heidelberg (2009)

13. Kass, M., Witkin, A., Terzopoulos, D.: Snakes: Active contour models. International Journal of Computer Vision 1(4), 321-331 (1988)

14. Kelley, D.E., Thaete, F.L., Troost, F., Huwe, T., Goodpaster, B.H.: Subdivisions of subcutaneous abdominal adipose tissue and insulin resistance. The American Journal of Physiology - Endocrinology and Metabolism 278, E941-E948 (2000)

15. Kullberg, J., Von Below, C., Lönn, L., Lind, L., Ahlström, H., Johansson, L.: Practical approach for estimation of subcutaneous and visceral adipose tissue. Clinical Physiology and Functional Imaging 27(3), 148-153 (2007)

16. Leinhard, O.D., Johansson, A., Rydell, J., Smedby, Ö., Nyström, F., Lundberg, P., Borga, M.: Quantitative abdominal fat estimation using mri. In: 19th International Conference on Pattern Recognition, ICPR 2008, pp. 1-4 (December 2008)

17. Positano, V., Gastaldelli, A., Sironi, A., Santarelli, M.F., Lombardi, M., Landini, L.: An accurate and robust method for unsupervised assessment of abdominal fat by MRI. Journal of Magnetic Resonance Imaging 20(4), 684-689 (2004)

18. Sled, J.G., Zijdenbos, A.P., Evans, A.C.: A nonparametric method for automatic correction of intensitynonuniformity in MRI data. IEEE Transactions on Medical Imaging 17, 87-97 (1998)

19. Wild, S., Roglic, G., Green, A., Sicree, R., King, H.: Global prevalence of diabetes. Diabetes Care 27(5), 1047 (2004)

20. Xu, C., Pham, D.L., Prince, J.L.: Handbook of Medical Imaging. Medical Image Processing and Analysis, ch. 3, vol. 2. SPIE Press, Bellingham (2000) 